\title{
INSTABILITY THROUGH POROUS MEDIUM OF TWO VISCOUS SUPERPOSED CONDUCTING FLUIDS
}

\author{
R.C. SHARMA and K.P. THAKUR \\ Department of Mathematics, Himachal Pradesh University, Simla-171005, India. \\ (Received July 11, 1979 and in revised form December 10, 1980)
}

ABSTRACT. The stability of the plane interface separating two viscous superposed conducting fluids through porous medium is studied when the whole system is immersed in a uniform horizontal magnetic field. The stability analysis is carried out for two highly viscous fluids of equal kinematic viscosities, for mathematical simplicity. It is found that the stability criterion is independent of the effects of viscosity and porosity of the medium and is dependent on the orientation and magnitude of the magnetic field. The magnetic field is found to stabilize a certain wave number range of the unstable configuration. The behaviour of growth rates with respect to viscosity, porosity and medium permeability are examined analytically.

KEY WORDS AND PHRASES. Instability, porous medium, conducting fluids. 1980 MATHEMATICS SUBJECT CLASSIFICATION CODES. 76E25, 76005

1. INTRODUCTION.

The instability of the plane interface between two fluids, under varying assumptions of hydrodynamics and hydromagnetics, has been discussed by Chandrasekhar [1]. Bhatia [2] has studied the influence of viscosity on the stability of the plane interface separating two incompressible superposed conducting fluids of 
uniform densities, when the whole system is immersed in a uniform horizontal magnetic field. He has carried out the stability analysis for two highly viscous fluids of equal kinematic viscosities and different uniform densities. When the fluid slowly percolates through the pores of the rock, the gross effect is represented by Darcy's law which states that the usual viscous term in the equations of fluid motion is replaced by the resistance term $\left(\mu / k_{1}\right) \vec{q}$, where $\mu$ is the viscosity of the fluid, $k_{1}$ the permeability of the medium and $\vec{q}$ the velocity of the fluid. Wooding [3] has experimentally observed, in the absence of viscous dissipation and considering only Darcy resistance, that convection sets on as a fairly regular cellular pattern in the horizontal. This problem in the case of a conducting fluid considering both Darcy and viscous resistances has been investigated by Prabhamani and Rudraiah [4]. Saville [5] has studied the stability of motions involving fluid interfaces in porous media. Various problems of fluid flows through porous medium have been treated by Saffman and Taylor [6], Chouke et al [7], Scheidegger [8], Yih [9], Nayfeh [10] and Rudraiah and Prabhamani [11].

The instability of two viscous superposed conducting fluids through porous medium may find applications in geophysics. It is therefore the motivation of this study to examine the effects of viscosity and medium permeability on the stability of the plane interface separating two incompressible superposed conducting fluids of uniform densities, when the whole system is immersed in a uniform horizontal magnetic field. We examine the roles of viscosity, medium permeability and magnetic field on the instability problem. This aspect forms the subject matter of the present study wherein we have carried out the stability analysis for two highly viscous fluids of equal kinematic viscosities and different uniform densities.

\section{PERTURBATION EQUATIONS.}

Consider the motion of an incompressible, infinitely conducting viscous fluid (of variable viscosity $\mu_{0}(z)$ ) in the presence of a uniform magnetic field $\left.\vec{H}_{(}, H_{x}, 0\right)$. Let $\vec{q}(u, v, w), \delta \rho, \delta p$ and $\vec{h}\left(h_{x}, h_{y}, h_{z}\right)$ denote the perturbations in velocity, density $\rho$, pressure $\mathrm{p}$ and magnetic field $\overrightarrow{\mathrm{H}}$ respectively. Then the linearized perturbation equations of a fluid flowing throughaporous medium when both Darcy as well as viscous resistances are present are: 
$\frac{\rho}{\varepsilon} \frac{\partial \vec{q}}{\partial t}=-\nabla \delta p+\vec{g} \delta \rho+\frac{1}{4 \pi}(\nabla \times \vec{h}) \times \vec{H}-\frac{\rho \nu}{k_{1}} \vec{q}+\frac{1}{\varepsilon}\left[\mu \nabla^{2} \vec{q}+(\nabla \vec{q}) \cdot \nabla \mu+(\nabla \mu \cdot \nabla) \vec{q}\right]$,

$\nabla \cdot \vec{q}=0, \quad \nabla \cdot \vec{h}=0$,

$\varepsilon \frac{\partial \vec{h}}{\partial t}=\nabla \times(\vec{q} \times \vec{H})$

$\varepsilon \frac{\partial}{\partial t} \delta \rho+(\vec{q} \cdot \nabla) \rho=0$

Equation (4) ensures that the density of every particle remains unchanged as we follow it with its motion. $\nu(=\mu / \rho)$ denotes the kinematic viscosity of the fluid, $\varepsilon$ is porosity $(0<\varepsilon<1)$ and $\vec{g}(0,0,-g)$ is the acceleration due to gravity. $\varepsilon \rightarrow 1$ and $\mathrm{k}_{1} \rightarrow \infty$ correspond to nonporous medium. Analyzing the disturbances into normal modes, we assume that the perturbed quantities have the space $(x, y, z)$ and time ( $t$ ) dependence of the form

$f(z) \exp \left(i k_{x} x+i k_{y} y+n t\right)$

where $k_{x}, k_{y}$ are horizontal wave numbers $\left(k^{2}=k_{x}^{2}+k_{y}^{2}\right), n$ is the growth rate of the harmonic disturbance and $f(z)$ is some function of $z$.

For perturbations of the form (2.5), equations (2.1)-(2.4) give

$\rho\left(\frac{n}{\varepsilon}+\frac{\nu}{k_{1}}\right) u+\frac{H}{4 \pi}\left(i k_{x} h_{y}-i k_{y} h_{x}\right)=-i k_{x} \delta p+\frac{1}{\varepsilon}\left[\mu\left(D^{2}-k^{2}\right) u+(D \mu)\left(i k_{y} w+D u\right)\right]$,
$\rho\left(\frac{n}{\varepsilon}+\frac{\nu}{k_{1}}\right) v+\frac{H_{x}}{4 \pi}\left(i k_{y} h_{x}-i k_{x} h_{y}\right)=-i k_{y} \delta p+\frac{1}{\varepsilon}\left[\mu\left(D^{2}-k^{2}\right) v+(D \mu)\left(i k_{y} w+D v\right)\right]$,
$\rho\left(\frac{n}{\varepsilon}+\frac{\nu}{k_{1}}\right) w+\frac{H^{\prime}}{4 \pi}\left(D h_{x}-i k_{x} h_{z}\right)+\frac{H_{y}}{4 \pi}\left(D h_{y}-i k_{y} h_{z}\right)=-D \delta p-g \delta \rho+\frac{1}{\varepsilon}\left[\mu\left(D^{2}-k^{2}\right) w+2(D \mu)(D w)\right]$,

$i k_{x} u+i k_{y} v+D w=0, \quad i k_{x} h_{x}+i k_{y} h_{y}+D h_{z}=0$

$\mathrm{n} \delta \rho=-\mathrm{w}(\mathrm{D} \rho) / \varepsilon$,

$n \vec{h}=\left(i k_{x} H_{x}+i k_{y} H_{y}\right) \vec{q} / \varepsilon$,

where $D=d / d z$. 
Multiplying (2.6) and (2.7) by $-i k_{x}$ and $-i k_{y}$ respectively and adding, using (2.9)(2.11) and finally, eliminating $\delta p$ between the resulting equation and (2.8), we obtain the equation in w:

$\left[k^{2} \rho\left(\frac{\mathrm{n}}{\varepsilon}+\frac{\nu}{k_{1}}\right) w-D\left\{\rho\left(\frac{n}{\varepsilon}+\frac{\nu}{k_{1}}\right) D w\right\}\right]-\frac{g k^{2}}{n \varepsilon}(D \rho) w-\frac{1}{4 \pi n \varepsilon}\left(k_{x} H_{x}+k_{y} H_{y}\right)^{2}\left(D^{2}-k^{2}\right) w$

$+\frac{1}{\varepsilon}\left[\mu\left(D^{2}-k^{2}\right)^{2} w+2(D \mu)\left(D^{2}-k^{2}\right) D w+\left(D^{2} \mu\right)\left(D^{2}+k^{2}\right) w\right]=0$.

\section{TWO SUPERPOSED VISCOUS FLUIDS OF UNIFORM DENSITIES.}

Here we consider the case when two superposed fluids of uniform densities $\rho_{1}$ and $\rho_{2}$ and uniform viscosities $\mu_{1}$ and $\mu_{2}$ are separated by a horizontal boundary at $z=0$. The subscripts 1 and 2 distinguish the lower and upper fluids respectively. Then, in each region of constant $\rho_{1}, \mu_{1}$ and constant $\rho_{2}, \mu_{2}$, equation (2.12) becomes $\left(D^{2}-k^{2}\right)\left(D^{2}-K^{2}\right) w=0$

where

$\mathrm{K}^{2}=\mathrm{k}^{2}+\frac{\mathrm{n}}{\nu}\left\{1+\frac{\nu \varepsilon}{\mathrm{nk}}+\frac{1}{4 \pi \mathrm{n}^{2} \rho}\left(\mathrm{k}_{\mathrm{x}} \mathrm{H} \mathrm{x}_{\mathrm{y}}+\mathrm{k}_{\mathrm{y}} \mathrm{H}^{2}\right\}\right.$

Since $w$ must vanish both when $z \rightarrow-\infty$ (in the lower fluid) and $z \rightarrow+\infty$ (in the upper fluid), the solutions appropriate to the two regions can be written as

$w_{1}=A_{1} e^{+k z}+B_{1} e^{+K_{1} z} \quad(z<0)$,
$w_{2}=A_{2} e^{-k z}+B_{2} e^{-K_{2} z} \quad(z>0)$,

where $A_{1}, B_{1}, A_{2}, B_{2}$ are constants,

$\mathrm{k}_{1}=\sqrt{\mathrm{k}^{2}+\frac{\mathrm{n}}{v_{1}}\left\{1+\frac{\nu_{1} \varepsilon}{\mathrm{nk}}+\frac{1}{4 \pi \mathrm{n}^{2} \rho_{1}}\left(\mathrm{k}_{\mathrm{x}} \mathrm{H}_{\mathrm{x}}+\mathrm{k}_{\mathrm{y}} \mathrm{H}_{\mathrm{y}}\right)^{2}\right\}}$

and

$\mathrm{K}_{2}=\sqrt{\mathrm{k}^{2}+\frac{\mathrm{n}}{v_{2}}\left\{1+\frac{v_{2} \varepsilon}{\mathrm{nk}_{1}}+\frac{1}{4 \pi \mathrm{n}^{2} \rho_{2}}\left(\mathrm{k}_{\mathrm{x}} \mathrm{H}_{\mathrm{x}}+\mathrm{k}_{\mathrm{y}} \mathrm{H}_{\mathrm{y}}\right)^{2}\right\}}$. 
In writing the solutions (3.3) and (3.4) it is assumed that $K_{1}$ and $K_{2}$ are so defined that their real parts are positive. The solutions (3.3) and (3.4) must satisfy certain boundary conditions. The boundary conditions to be satisfied at the interface $z=0$ are (Chandrasekhar [1], p.432)

w,

Dw,

and

$\mu\left(D^{2}+k^{2}\right) w$,

must be continuous.

Integrating (2.12) across the interface $z=0$, we obtain another condition $\left\{\rho_{2}\left(\frac{\mathrm{n}}{\varepsilon}+\frac{\nu_{2}}{\mathrm{k}_{1}}\right) D w_{2}-\frac{\mu_{2}}{\varepsilon}\left(D^{2}-k^{2}\right) D w_{2}+\frac{1}{4 \pi n \varepsilon}\left(k_{x} H_{x}+k_{y} H_{y}\right)^{2} D w_{2}\right\}_{z=0}$

$-\left\{\rho_{1}\left(\frac{n}{\varepsilon}+\frac{\nu_{1}}{k_{1}}\right) D w_{1}-\frac{\mu_{1}}{\varepsilon}\left(D^{2}-k^{2}\right) D w_{1}+\frac{1}{4 \pi n \varepsilon}\left(k_{x} H_{x}+k_{y} H_{y}\right)^{2} D_{1} w_{z=0}\right.$

$=-\frac{g k^{2}}{n \varepsilon}\left(\rho_{2}-\rho_{1}\right) w_{0}-\frac{2 k^{2}}{\varepsilon}\left(\mu_{2}-\mu_{1}\right)(D w)_{0}$

where $w_{0}$ and $(D w)_{0}$ are the unique values of these quantities at $z=0$.

Applying the conditions (3.7)-(3.10) to the solutions (3.3) and (3.4), we obtain

$$
\begin{aligned}
& \mathrm{A}_{1}+\mathrm{B}_{1}=\mathrm{A}_{2}+\mathrm{B}_{2}, \\
& \mathrm{kA}_{1}+\mathrm{K}_{1} \mathrm{~B}_{1}=-\mathrm{kA}_{2}-\mathrm{K}_{2} \mathrm{~B}_{2}, \\
& \mu_{1}\left\{2 \mathrm{k}^{2} \mathrm{~A}_{1}+\left(\mathrm{K}_{1}^{2}+\mathrm{k}^{2}\right) \mathrm{B}_{1}\right\}=\mu_{2}\left\{2 \mathrm{k}^{2} \mathrm{~A}_{2}+\left(\mathrm{K}_{2}{ }^{2}+\mathrm{k}^{2}\right) \mathrm{B}_{2}\right\} \\
& -\mathrm{k} \rho_{2}\left(\frac{1}{\varepsilon}+\frac{\nu_{2}}{\mathrm{nk}}\right) \mathrm{A}_{2}-\mathrm{k}_{1}\left(\frac{1}{\varepsilon}+\frac{\nu_{1}}{\mathrm{nk}}\right) \mathrm{A}_{1}-\left(\mathrm{kA}_{1}+\mathrm{kA}_{2}\right) \frac{1}{4 \pi \mathrm{n}^{2} \varepsilon}\left(\mathrm{k}_{\mathrm{x}} \mathrm{H}_{\mathrm{x}}+\mathrm{k}_{\mathrm{y}} \mathrm{H}_{\mathrm{y}}\right)^{2} \\
& \quad=-\frac{\mathrm{gk}{ }^{2}}{2 \mathrm{n}^{2} \varepsilon}\left(\rho_{2}-\rho_{1}\right)\left(\mathrm{A}_{1}+\mathrm{B}_{1}+\mathrm{A}_{2}+\mathrm{B}_{2}\right)+\frac{\mathrm{k}^{2}}{\mathrm{n} \varepsilon}\left(\mu_{1}-\mu_{2}\right)\left(\mathrm{kA}_{1}+\mathrm{K}_{1} \mathrm{~B}_{1}-\mathrm{kA}_{2}-\mathrm{K}_{2} \mathrm{~B}_{2}\right) .
\end{aligned}
$$


370

R.C. SHARIA AND K.P. THAKUR

Eliminating the constants $\mathrm{A}_{1}, \mathrm{~A}_{2}, \mathrm{~B}_{1}, \mathrm{~B}_{2}$ from $(3.11)-(3.14)$, we obtain

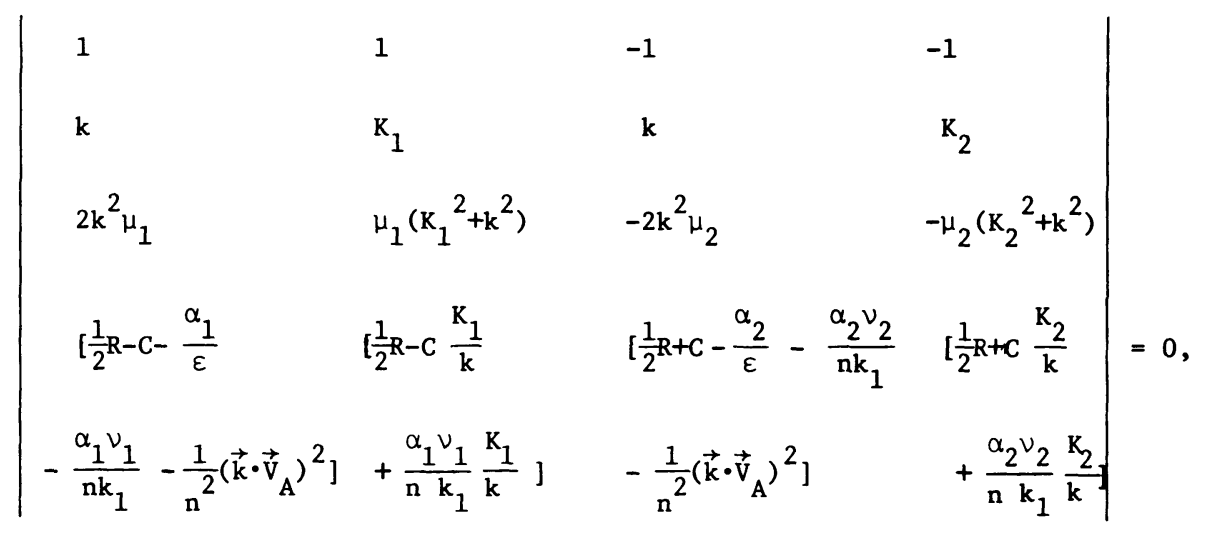

where

$$
\begin{aligned}
& \alpha_{1,2}=\frac{\rho_{1,2}}{\rho_{1}+\rho_{2}}, \quad\left(\vec{k} \cdot \vec{v}_{A}\right)^{2}=\frac{\left(k_{x} H_{x}+k_{y} H_{y}\right)^{2}}{4 \pi\left(\rho_{1}+\rho_{2}\right) \varepsilon} . \\
& R=\frac{g k}{\varepsilon n^{2}}\left(\alpha_{2}-\alpha_{1}\right), \quad C=\frac{k^{2}}{n \varepsilon} \frac{\mu_{1}-\mu_{2}}{\rho_{1}+\rho_{2}}=\frac{k^{2}}{n \varepsilon}\left(\alpha_{1} \nu_{1}-\alpha_{2} \nu_{2}\right), \\
& \text { and } \vec{v}_{A} \text { is the Alfvén velocity vector. }
\end{aligned}
$$

Evaluating the determinant (3.15), we obtain the following characteristic equation

$$
\begin{aligned}
& \left(k_{1}-k\right)\left[2 k^{2}\left(\alpha_{1} \nu_{1}-\alpha_{2} \nu_{2}\right) \quad\left\{\frac{C}{k}\left(k_{2}-k\right)+\alpha_{2}\left(\frac{1}{\varepsilon}+\frac{\nu_{2}}{n k_{1}}\right)+\frac{1}{n^{2}}\left(\vec{k} \cdot \vec{v}_{A}\right)^{2}\right\}\right. \\
& \left.+\left\{\alpha_{2} n+\frac{\alpha_{2} \nu_{2} \varepsilon}{k_{1}}+\frac{\varepsilon}{n}\left(\vec{k} \cdot \vec{v}_{A}\right)^{2}\right\}\left\{R-\frac{1}{\varepsilon}-\frac{\left(\alpha_{1} \nu_{1}+\alpha_{2} \nu_{2}\right)}{n_{1}}-\frac{2}{n^{2}}\left(\vec{k}_{1} \cdot \vec{v}_{A}\right)^{2}\right\}\right] \\
& -2 \mathrm{k}\left[\left\{\alpha_{1} \mathrm{n}+\frac{\alpha_{1} \nu_{1} \varepsilon}{\mathrm{k}_{1}}+\frac{\varepsilon}{\mathrm{n}}\left(\overrightarrow{\mathrm{k}}_{\mathrm{v}} \cdot \overrightarrow{\mathrm{v}}_{\mathrm{A}}\right)^{2}\right\}\left\{\alpha_{2}\left(\frac{1}{\varepsilon}+\frac{\nu_{2}}{\mathrm{nk}_{1}}\right)+\frac{\mathrm{c}}{\mathrm{k}}\left(\mathrm{k}_{2}-\mathrm{k}\right)+\frac{1}{\mathrm{n}^{2}}\left(\overrightarrow{\mathrm{k}}_{\mathrm{v}} \cdot \overrightarrow{\mathrm{v}}_{\mathrm{A}}\right)^{2}\right\}\right. \\
& \left.+\left\{\alpha_{2} n+\frac{\alpha_{2} \nu_{2} \varepsilon}{k_{1}}+\frac{\varepsilon}{n}\left(\vec{k}_{1} \cdot \vec{v}_{A}\right)^{2}\right\}\left\{\alpha_{1}\left(\frac{1}{\varepsilon}+\frac{\nu_{1}}{n k_{1}}\right)-\frac{C}{k}\left(k_{1}-k\right)+\frac{1}{n^{2}}\left(\vec{k} \cdot \vec{v}_{A}\right)^{2}\right\}\right] \\
& +\left(\mathrm{k}_{2}-\mathrm{k}\right)\left[\left\{\alpha_{1} \mathrm{n}+\frac{\alpha_{1} \nu_{1} \varepsilon}{\mathrm{k}_{1}}+\frac{\varepsilon}{\mathrm{n}}\left(\overrightarrow{\mathrm{k}} \cdot \overrightarrow{\mathrm{v}}_{\mathrm{A}}\right)^{2}\right\}\left\{\mathrm{R}-\frac{1}{\varepsilon}-\frac{\alpha_{1} \nu_{1}+\alpha_{2} \nu_{2}}{\mathrm{nk}_{1}}-\frac{2}{\mathrm{n}^{2}}\left(\overrightarrow{\mathrm{k}}_{\mathrm{v}} \cdot \overrightarrow{\mathrm{v}}_{\mathrm{A}}\right)^{2}\right\}\right. \\
& \left.-2 \mathrm{k}^{2}\left(\alpha_{1} \nu_{1}-\alpha_{2} \nu_{2}\right)\left\{\alpha_{1}\left(\frac{1}{\varepsilon}+\frac{\nu_{1}}{n k_{1}}\right)-\frac{c}{k} \quad\left(k_{1}-k\right)+\frac{1}{n^{2}}\left(\vec{k}_{1} \cdot \vec{v}_{A}\right)^{2}\right\}\right]=0 .
\end{aligned}
$$


Since $K_{1}$ and $K_{2}$ involve square roots, the dispersion relation (3.16) is quite complex. We therefore carry out the stability analysis for highly viscous fluids. Under this assumption of highly viscous fluids, we have

$$
\begin{aligned}
\mathrm{K} & =\mathrm{k}\left[1+\frac{\mathrm{n}}{\nu^{2}}+\frac{\varepsilon}{\mathrm{k}^{2} \mathrm{k}_{1}}+\frac{\varepsilon\left(\mathrm{k}_{\mathrm{x}} \mathrm{H}+\mathrm{k}_{\mathrm{y}} \mathrm{H}_{\mathrm{y}}\right)^{2}}{4 \pi \rho v \mathrm{nk}^{2}}\right]^{\frac{1}{2}} \\
& =\mathrm{k}\left[1+\frac{\mathrm{n}}{2 \nu \mathrm{k}^{2}}+\frac{\varepsilon}{2 \mathrm{k}^{2} \mathrm{k}_{1}}+\frac{\varepsilon\left(\rho_{1}+\rho_{2}\right)}{2 \mathrm{nk}^{2} \rho \nu}\left(\overrightarrow{\mathrm{k}}_{\mathrm{v}} \cdot \overrightarrow{\mathrm{v}}_{\mathrm{A}}\right)^{2}\right],
\end{aligned}
$$

so that

$\mathrm{K}_{1}-\mathrm{k}=\frac{\mathrm{n}}{2 \nu_{1} \mathrm{k}}+\frac{\varepsilon}{2 \mathrm{k} \mathrm{k}_{1}}+\frac{\varepsilon}{2 \mathrm{nk} \alpha_{1} \nu_{1}}\left(\overrightarrow{\mathrm{k}} \cdot \overrightarrow{\mathrm{v}}_{\mathrm{A}}\right)^{2}$

and

$\mathrm{K}_{2}-\mathrm{k}=\frac{\mathrm{n}}{2 \nu_{2} \mathrm{k}}+\frac{\varepsilon}{2 \mathrm{kk}{ }_{1}}+\frac{\varepsilon}{2 \mathrm{nk} \alpha_{2} \nu_{2}}\left(\overrightarrow{\mathrm{k}} \cdot \overrightarrow{\mathrm{V}}_{\mathrm{A}}\right)^{2}$

Substituting the values of $\mathrm{K}_{1}$ and $\mathrm{K}_{2}$ from (3.18), (3.19) in (3.16) and putting $v_{1}=v_{2}=v$ (the case of equal kinematic viscosities, for mathematical simplicity, as in Chandrasekhar [1]), we obtain the following dispersion relation

$$
\begin{aligned}
& \frac{1}{\varepsilon} n^{6}+\left[\frac{3 \nu}{k_{1}}+\frac{2 \alpha_{1} \alpha_{2}}{\varepsilon}+\left(\alpha_{1}-\alpha_{2}\right)^{2}\left(\frac{1}{2}+\frac{k^{2} v}{\varepsilon} \frac{}{2-\varepsilon}\right)\right] n^{5}+\left[\frac{\left(\vec{k} \cdot \vec{v}_{A}\right)^{2}}{\alpha_{1} \alpha_{2}}+A+\frac{3 \nu^{2} \varepsilon}{k_{1}^{2}}+\frac{4 k^{2} v^{2}}{k_{1}}\left(\alpha_{1}-\alpha_{2}\right)^{2} \times\right. \\
& \left.\times(1-\varepsilon)+\frac{\nu}{k_{1}}\left\{4 \alpha_{1} \alpha_{2}+\varepsilon\left(\alpha_{1}-\alpha_{2}\right)^{2}\right\}\right] n^{4}+\left[\frac { \nu \varepsilon } { k _ { 1 } } \left\{2 A+\frac{\nu^{2} \varepsilon}{k_{1}^{2}}+\frac{2 k^{2} \nu^{2}}{k_{1}}\left(\alpha_{1}-\alpha_{2}\right)^{2}(1-\varepsilon)+2 \alpha_{1} \alpha_{2} \nu+\frac{\nu \varepsilon}{2 k_{1}}\right.\right. \\
& \left.+\left(\vec{k} \cdot \vec{v}_{A}\right)^{2}\left\{2+\frac{\varepsilon}{2 \alpha_{1} \alpha_{2}}\left(\alpha_{1}-\alpha_{2}\right)^{2}+\frac{2 v \varepsilon}{k_{1} \alpha_{1} \alpha_{2}}+\frac{2 k^{2} v}{\alpha_{1} \alpha_{2}}\left(\alpha_{1}-\alpha_{2}\right)^{2}(1-\varepsilon)\right\}\right] n^{3} \\
& +\left[\frac{\varepsilon\left(\vec{k} \cdot \vec{v}_{A}\right)^{2}}{\alpha_{1} \alpha_{2}}\left\{A+\left(\vec{k} \cdot \vec{v}_{A}\right)^{2}+\frac{\nu^{2} \varepsilon}{k_{1}{ }^{2}}\left(\alpha_{1}{ }^{2}+\alpha_{2}{ }^{2}\right)+\frac{2 k^{2} v^{2}}{k_{1}}\left(\alpha_{1}-\alpha_{2}\right)^{2}(1-\varepsilon)\right\}\right. \\
& \left.+\frac{\nu \varepsilon}{k_{1}}\left\{2\left(\vec{k} \cdot \vec{v}_{A}\right)^{2}\left(1+\frac{\nu \varepsilon}{k_{1}}\right)+\frac{\nu \varepsilon A}{k_{1}}+\frac{\varepsilon}{2 \alpha_{1} \alpha_{2}}\left(\vec{k} \cdot \vec{v}_{A}\right)^{2}\left(\alpha_{1}-\alpha_{2}\right)^{2}\right\}\right] n^{2} \\
& +\left[\varepsilon\left(\vec{k} \cdot \vec{v}_{A}\right)^{4}\left\{2+\frac{\varepsilon}{2 \alpha_{1} \alpha_{2}}\left(\alpha_{1}-\alpha_{2}\right)^{2}\right\}+\frac{\varepsilon}{\alpha_{1} \alpha_{2}}\left(\vec{k} \cdot \vec{v}_{A}\right)^{2}\left\{\frac{\nu \varepsilon}{k_{1}}\left(\vec{k} \cdot \vec{v}_{A}\right)^{2}+\frac{\nu \varepsilon A}{k_{1}}\right.\right.
\end{aligned}
$$


$\left.\left.+\frac{2 k^{2} v\left(\alpha_{1}-\alpha_{2}\right)^{2}\left(\vec{k}_{0} \cdot \vec{v}_{A}\right)^{2}}{\alpha_{1} \alpha_{2}}(1-\varepsilon)\right\}\right] n+\frac{\varepsilon^{2} A}{\alpha_{1} \alpha_{2}}\left(\vec{k}^{2} \cdot \vec{v}_{A}\right)^{4}=0$,

where we have written $2\left(\vec{k} \cdot \vec{v}_{A}\right)^{2}+\frac{g k}{\varepsilon}\left(\alpha_{1}-\alpha_{2}\right)=A$.

\section{(i) Stable case}

For the potentially stable arrangement $\alpha_{1}>\alpha_{2}$, we find by applying Hurwitz' criterion to (3.20), that (as all the coefficients in (3.20) are then positive) all the roots of $\mathrm{n}$ are either real and negative or there are complex roots with negative real parts. The system is therefore stable in each case. The potentially stable configuration, therefore, remains stable whether the effects of viscosity and medium porosity are included or not.

\section{(ii) Unstable case}

For the potentially unstable arrangement $\alpha_{2}>\alpha_{1}$, the system is unstable in the hydrodynamic case for all wave numbers $k$ in the presence of viscosity effects and in the absence of porosity effects (Chandrasekhar [1]). Also the system, in the present case, is unstable if

$2\left(\overrightarrow{\mathrm{k}} \cdot \overrightarrow{\mathrm{v}}_{\mathrm{A}}\right)^{2}<\frac{\mathrm{gk}}{\varepsilon}\left(\alpha_{2}-\alpha_{1}\right)$

In the present hydromagnetic case we find, by applying Hurwitz' criterion to $(3.20)$ when $\alpha_{2}>\alpha_{1}$, that the system is stable for all wave numbers which satisfy the inequality

$2\left(\vec{k} \cdot \vec{v}_{A}\right)^{2}>\frac{g k}{\varepsilon}\left(\alpha_{2}-\alpha_{1}\right)$,

i.e. $2 k\left(v_{1} \cos \theta+v_{2} \sin \theta\right)^{2}>\frac{g}{\varepsilon}\left(\alpha_{2}-\alpha_{1}\right)$,

where $v_{1}$ and $v_{2}$ are the Alfven velocities in the $x$ and $y$ directions and $\theta$ is the angle between $\vec{k}$ and $H_{x}$.

The stability criterion (3.22) is independent of the effects of viscosity and medium porosity. The magnetic field stabilizes a certain wave number range $k>k$ * where 
$k *=g\left(\alpha_{2}-\alpha_{1}\right) / 2 \varepsilon\left(v_{1} \cos \theta+v_{2} \sin \theta\right)^{2}$,

of the unstable configuration even in the presence of the effects of viscosity and medium porosity. The critical wave number $k^{*}$, above which the system is stabilized, is dependent on the magnitudes $v_{1}$ and $v_{2}$ of the magnetic field as well as the orientation of the magnetic field $\theta$.

We now examine the behaviour of growth rates with respect to viscosity, porosity and medium permeability analytically. Since for $\alpha_{2}>\alpha_{1}$ and $2\left(\vec{k}_{1} \cdot \vec{v}_{A}\right)^{2}<$ $\frac{\mathrm{gk}}{\varepsilon}\left(\alpha_{2}-\alpha_{1}\right),(3.20)$ has one positive root, let $\mathrm{n}_{\mathrm{o}}$ denote the positive root. Then (3.20) is satisfied if $\mathrm{n}_{0}$ is substituted in place of $\mathrm{n}$. To study the behaviour of growth rates with respect to viscosity and medium permeability, we examine the natures of $\mathrm{dn}_{0} / \mathrm{d} v$ and $\mathrm{dn}_{0} / \mathrm{dk}_{1}$. It follows that

$$
\begin{aligned}
& \left(6 A_{6} n_{o}^{5}+5 A_{5} n_{o}^{4}+4 A_{4} n_{o}^{3}+3 A_{3} n_{o}^{2}+2 A_{2} n_{o}+A_{1}\right) \frac{d n_{o}}{d k_{1}} \\
& =\frac{3 \nu}{\mathrm{k}_{1}{ }^{2}} \mathrm{n}_{\mathrm{o}}^{5}+\frac{1}{\mathrm{k}_{1}{ }^{2}}\left\{\frac{6 \nu^{2} \varepsilon}{\mathrm{k}_{1}}+4 \mathrm{k}^{2} \nu^{2}\left(\alpha_{1}-\alpha_{2}\right)^{2}(1-\varepsilon)+\nu\left\{4 \alpha_{1} \alpha_{2}+\varepsilon\left(\alpha_{1}-\alpha_{2}\right)^{2}\right\} \mathrm{n}_{\mathrm{o}}{ }^{4}\right. \\
& +\frac{1}{k_{1}{ }^{2}}\left\{\frac{2 \nu \varepsilon}{\alpha_{1} \alpha_{2}}\left(\vec{k} \cdot \vec{v}_{A}\right)^{2}-2 A v \varepsilon+\frac{3 \nu^{3} \varepsilon^{2}}{k_{1}{ }^{2}}+\frac{4 k^{2} v^{3} \varepsilon\left(\alpha_{1}-\alpha_{2}\right)^{2}(1-\varepsilon)}{k_{1}}+2 \alpha_{1} \alpha_{2} \nu^{2} \varepsilon+\frac{\nu^{2} \varepsilon^{2}}{k_{1}}\right\} n_{0}^{3} \\
& +\left[\frac{2 \nu^{2} \varepsilon\left(\vec{k}^{2} \cdot \vec{v}_{A}\right)^{2}}{\alpha_{1} \alpha_{2} k_{1}^{2}}\left\{\frac{\varepsilon\left(\alpha_{1}{ }^{2}+\alpha_{2}{ }^{2}\right)}{k_{1}}+k^{2}\left(\alpha_{1}-\alpha_{2}\right)^{2}(1-\varepsilon)\right\}+2\left(\vec{k}_{1} \cdot \vec{v}_{A}\right)^{2} \frac{v \varepsilon}{k_{1}{ }^{2}}\left(1+\frac{2 \nu \varepsilon}{k_{1}}\right)\right. \\
& \left.-\frac{2 v^{2} \varepsilon^{2} \mathrm{~A}}{\mathrm{k}_{1}{ }^{3}}+\frac{v \varepsilon^{2}}{2 \alpha_{1} \alpha_{2} \mathrm{k}_{1}^{2}}\left(\overrightarrow{\mathrm{k}} \cdot \overrightarrow{\mathrm{v}}_{\mathrm{A}}\right)^{2}\left(\alpha_{1}-\alpha_{2}\right)^{2}\right] \mathrm{n}_{\mathrm{o}}^{2} \\
& +\left[\frac{\varepsilon}{\alpha_{1} \alpha_{2} k_{1}^{2}}\left(\vec{k} \cdot \vec{v}_{A}\right)^{2}\left\{v \varepsilon\left(\vec{k} \cdot \vec{v}_{A}\right)^{2}-v \varepsilon A\right\}\right] n_{0}
\end{aligned}
$$

and

$$
-\left(6 A_{6} n_{0}^{5}+5 A_{5} n_{0}^{4}+4 A_{4} n_{0}^{3}+3 A_{3} n_{0}^{2}+2 A_{2} n_{0}+A_{1}\right) \frac{d n_{o}}{d \nu}=
$$




$$
\begin{aligned}
& =\left[\frac{3}{\mathrm{k}_{1}}+\frac{\mathrm{k}^{2}}{\varepsilon}(2-\varepsilon)\left(\alpha_{1}-\alpha_{2}\right)^{2}\right] \mathrm{n}_{\mathrm{o}}^{5}+\left[\frac{6 v \varepsilon}{\mathrm{k}_{1}{ }^{2}}+\frac{8 v \mathrm{k}^{2}}{\mathrm{k}_{1}}\left(\alpha_{1}-\alpha_{2}\right)^{2}(1-\varepsilon)+\frac{1}{\mathrm{k}_{1}}\left\{4 \alpha_{1} \alpha_{2}+\varepsilon\left(\alpha_{1}-\alpha_{2}\right)^{2}\right\}\right] \mathrm{n}_{\mathrm{o}}{ }^{4} \\
& +\left[\frac{3 \varepsilon^{2} \nu^{2}}{\mathrm{k}_{1}{ }^{2}}-\frac{2 \mathrm{~A} \varepsilon}{\mathrm{k}_{1}}+\frac{6 \mathrm{k}^{2} \varepsilon \nu^{2}}{\mathrm{k}_{1}{ }^{2}}\left(\alpha_{1}-\alpha_{2}\right)^{2}(1-\varepsilon)+\frac{4 \alpha_{1} \alpha_{2} \varepsilon \nu}{\mathrm{k}_{1}}+\frac{v \varepsilon^{2}}{\mathrm{k}_{1}{ }^{2}}+\frac{2 \varepsilon\left(\overrightarrow{\mathrm{k}}_{\mathrm{v}} \cdot \overrightarrow{\mathrm{v}}_{\mathrm{A}}\right)^{2}}{\alpha_{1} \alpha_{2} \mathrm{k}_{1}}\right. \\
& \left.+\frac{2 \mathrm{k}^{2}\left(\alpha_{1}-\alpha_{2}\right)^{2}(1-\varepsilon)}{\alpha_{1} \alpha_{2}}\left(\overrightarrow{\mathrm{k}} \cdot \overrightarrow{\mathrm{v}}_{\mathrm{A}}\right)^{2}\right] \mathrm{n}_{\mathrm{o}}^{3} \\
& +\frac{2 v \varepsilon^{2}\left(\vec{k} \cdot \vec{v}_{A}\right)^{2}}{\alpha_{1} \alpha_{2} k_{1}^{2}}\left(\alpha_{1}{ }^{2}+\alpha_{2}{ }^{2}\right)+\frac{4 \nu k^{2} \varepsilon\left(\vec{k} \cdot \vec{v}_{A}\right)^{2}}{\alpha_{1} \alpha_{2} k_{1}}\left(\alpha_{1}-\alpha_{2}\right)^{2}(1-\varepsilon)+2\left(\vec{k}_{1} \cdot \vec{v}_{A}\right)^{2} \frac{\varepsilon}{k_{1}} \\
& \left.+\frac{4 v \varepsilon^{2}}{\mathrm{k}_{1}{ }^{2}}\left(\overrightarrow{\mathrm{k}} \cdot \overrightarrow{\mathrm{v}}_{\mathrm{A}}\right)^{2}-\frac{2 v \varepsilon^{2} \mathrm{~A}}{\mathrm{k}_{1}{ }^{2}}+\frac{\varepsilon^{2}}{2 \mathrm{k}_{1} \alpha_{1} \alpha_{2}}\left(\overrightarrow{\mathrm{k}}^{2} \cdot \overrightarrow{\mathrm{v}}_{\mathrm{A}}\right)^{2}\left(\alpha_{1}-\alpha_{2}\right)^{2}\right] \mathrm{n}_{\mathrm{o}}{ }^{2} \\
& +\left[\frac{\varepsilon^{2}}{\alpha_{1} \alpha_{2} k_{1}}\left(\vec{k} \cdot \vec{v}_{A}\right)^{4}-\frac{\varepsilon^{2} A}{\alpha_{1} \alpha_{2} k_{1}}\left(\vec{k} \cdot \vec{v}_{A}\right)^{2}+\frac{2 k^{2} \varepsilon}{\alpha_{1}{ }^{2} \alpha_{2}{ }^{2}}\left(\vec{k} \cdot \vec{v}_{A}\right)^{4}\left(\alpha_{1}-\alpha_{2}\right)^{2}(1-\varepsilon)\right] n_{0},
\end{aligned}
$$

where $A_{5}, A_{4}, A_{3}, A_{2}$ and $A_{1}$ are the coefficients of $n_{0}^{5}, n_{0}^{4}, n_{0}^{3}, n_{0}^{2}$ and $n_{0}$ respectively in (3.20) where $n_{0}$ is substituted for $n$. It is evident from (3.24) and (3.25) that $\mathrm{dn}_{0} / \mathrm{dk}_{1}$ and $\mathrm{dn}_{\mathrm{o}} / \mathrm{d} \nu$ may be both positive or negative. Similarly it can be shown that $\mathrm{dn}_{\mathrm{o}} / \mathrm{d} \varepsilon$ may be both positive or negative. Thus the growth rates both increase or decrease with the increase in viscosity, porosity, and medium permeability. The viscosity, porosity, and medium permeability therefore have both stabilizing as well as destabilizing tendencies on the growth rates.

\section{REFERENCES}

1. CHANDRASEKHAR, S. Hydrodynamic and Hydromagnetic Stability. Oxford University Press, London, Chap. X, 1961.

2. BHATIA, P.K. Rayleigh-Taylor instability of two viscous superposed conducting fluids, Nuovo Cimento 19B, 161-168, 1974.

3. WOODING, R.A. Rayleigh instability of a thermal boundary layer in flow through a porous medium, J. Fluid Mech. 9, 183-192, 1960.

4. PRABHAMANI, P.R. and RUDRAIAH, N. Stability of hydromagnetic thermoconvective flow through porous medium, J. Appl. Mech. Ser. E. 40, 879-884, 1973.

5. SAVILLE, D.A. Stability of motions involving fluid interfaces in porous media, Phys. Fluids 12, 2438-2440, 1969. 
6. SAFFMAN, P.G. and TAYLOR, G.I. The penetration of a fluid into a porous medium or Hele-Shaw cell containing a more viscous liquid, Proc. Roy. Soc. A245, 321-329, 1958.

7. CHOUKE, R.L., VAN MEURS, P. and VAN DER POEL, C. The instability of slow, immiscible, viscous liquid-liquid displacement in permeable media, Trans. AIME 216, 188-194, 1959 .

8. SCHEIDEgGER, A.E. The Physics of Flow through Porous Media. University of Toronto Press, Macmillan, 1960.

9. YIH, C.S. Stratified Flows. Academic Press, 1980.

10. NAYFEH, A.H. Stability of liquid interfaces in porous media, Phys. Fluids 15 , 1751-1754, 1972.

11. RUDRAIAH, N. and PRABHAMANI, P.R. Thermal diffusion and convective stability of a two component fluid in a porous medium, 5 th International Heat Transfer Conference, Tokyo, Japan, CT 3.1, 79-82, 1974.

Present Address of the first author:

Dr. R.C. Sharma

Department of Physics

University of Alberta

Edmonton, Canada

T6G 2J1 


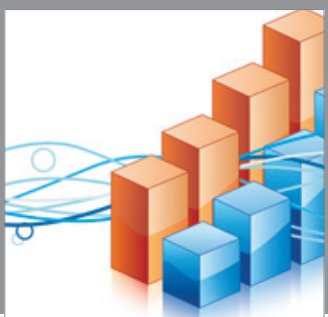

Advances in

Operations Research

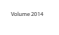

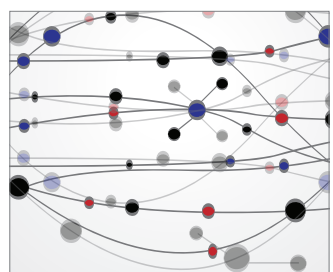

\section{The Scientific} World Journal
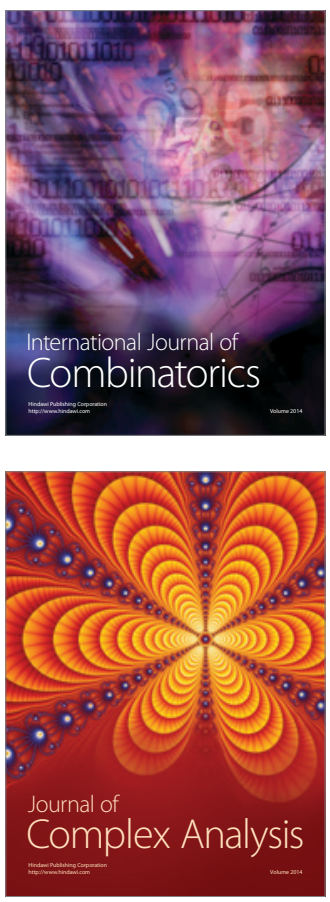

International Journal of

Mathematics and

Mathematical

Sciences
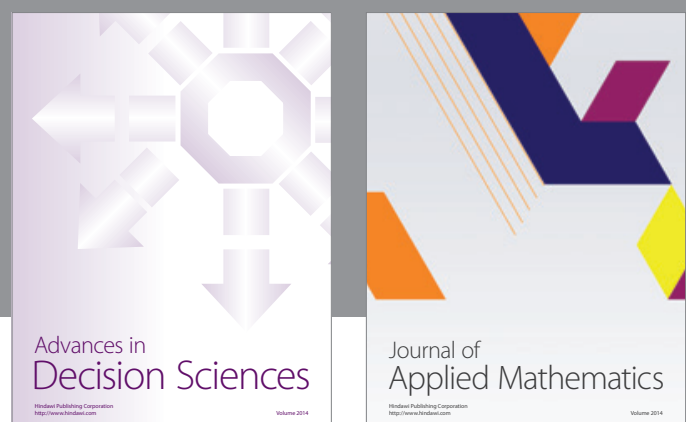

Journal of

Applied Mathematics
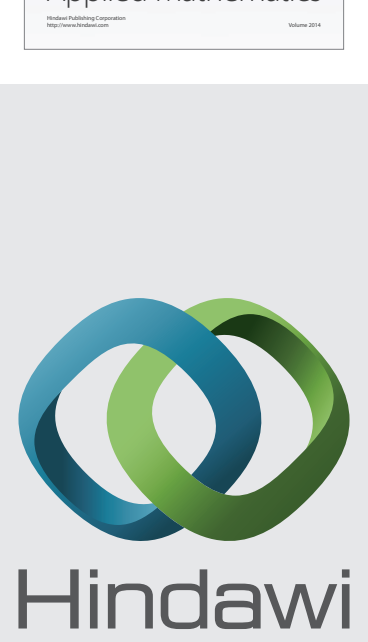

Submit your manuscripts at http://www.hindawi.com
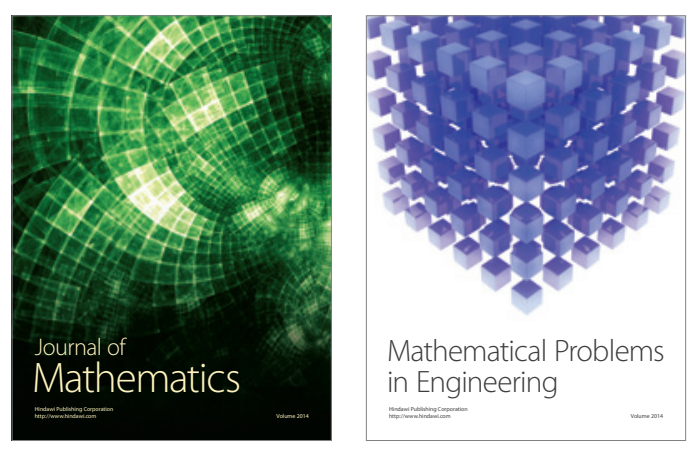

Mathematical Problems in Engineering
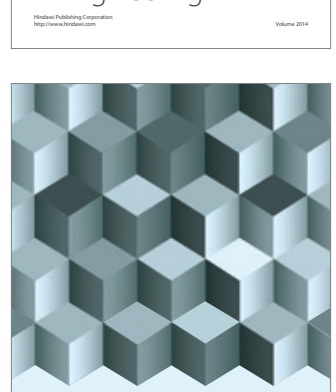

Journal of

Function Spaces
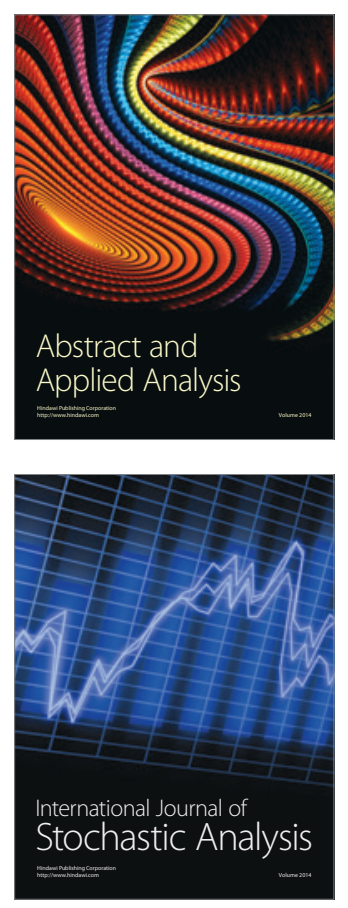

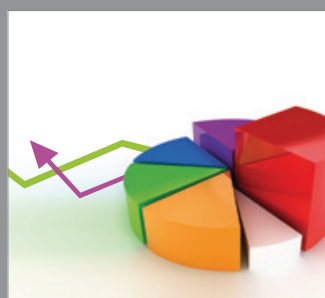

ournal of

Probability and Statistics

Promensencen
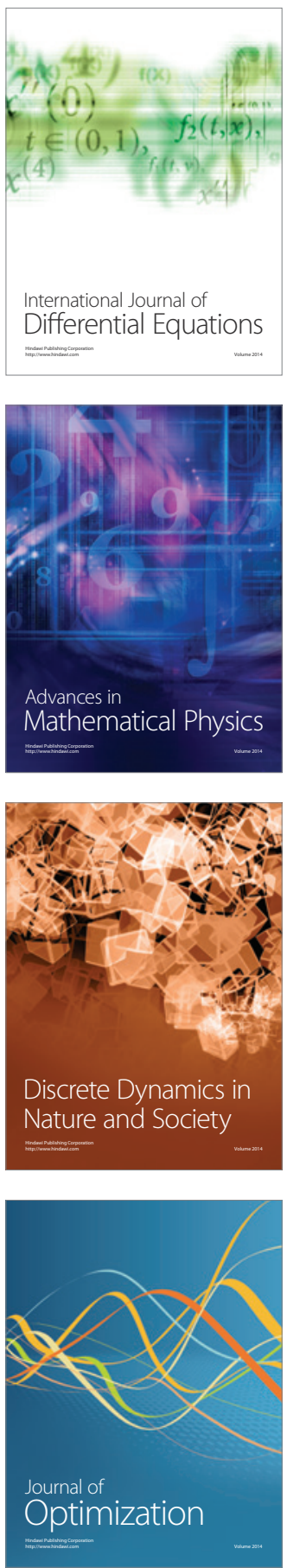